Original Paper http://ajol.info/index.php/ijbcs http://indexmedicus.afro.who.int

\title{
Aspects épidémiologiques et étiologiques des affections pulmonaires d'origine parasitaire et fongique en milieu hospitalier à Ouagadougou (Burkina Faso)
}

\author{
A.M. OUBAYYOU ${ }^{1 *}$, A. ZIDA ${ }^{2}$, H. MOUMOUNI ${ }^{3}$, A. SAVADOGO ${ }^{1}$, \\ A.S. TRAORE ${ }^{1}$ et M. OUEDRAOGO ${ }^{4}$ \\ ${ }^{1}$ Centre de Recherche en Sciences Biologiques Alimentaires et Nutritionnelles, Université de Ouagadougou, \\ 3 BP 7131, Ouagadougou 03, Burkina Faso. \\ ${ }^{2}$ Service de Parasitologie-Mycologie, Centre Hospitalier Universitaire Yalgado Ouédraogo, \\ 03 BP 7022, Ouagadougou 03, Burkina Faso. \\ ${ }^{3}$ Faculté des Sciences de la Santé, Université Abdou Moumouni de Niamey, BP: 131/125 Niamey-Niger. \\ ${ }^{4}$ Service de Pneumo-Phtisiologie du Centre Hospitalier Universitaire Yalgado Ouédraogo, 03 BP 7022, \\ Ouagadougou 03, Burkina Faso. \\ *Auteur correspondant ; E-mail : mamoudouabdoulaye92@yahoo.fr; Tel : (+227) 96191922
}

\section{RESUME}

Dans le souci de déterminer l'aspect épidémiologique et étiologique des affections pulmonaires d'origines parasitaire et fongique, une étude prospective transversale a été réalisée en milieu hospitalier à Ouagadougou de novembre 2012 en mai 2013, sur 103 patients suspectés avoir la maladie. Les examens parasitologiques, mycologiques et immunologiques ont été effectués selon les indications des prescripteurs, sur des prélèvements du liquide de lavage broncho-alvéolaire, du liquide pleural, des expectorations et du sang. A l'issue de ces examens, 59,2\% des patients confirmés porteurs de parasites et/ou de mycètes dans leur appareil pulmonaire avec une prédominance des cultivateurs $(26,2 \%)$ ont été détectés. Les hommes étaient les plus touchés avec un sex-ratio de 1,9. Parmi les antécédents médicaux, il y a eu une fréquence élevée des sujets à sérologie VIH positive. Trois parasites et 71 souches de champignons ont été isolés dont 4 types de coinfections fongiques. Ces données de bases montrent que les parasites et les champignons provoquent une pathologie pulmonaire non spécifique sur le plan clinique, radiologique et endoscopique. Le contexte épidémiologique et biologique permet d'orienter le diagnostic. La confirmation est apportée par la mise en évidence directe ou par des arguments indirects sérologiques. Ce qui permet d'obtenir une guérison par un traitement adapté au germe en cause.

() 2016 International Formulae Group. All rights reserved.

Mots clés : Affection pulmonaire, parasites, champignons, Burkina Faso.

\section{Epidemiological and etiological of lung diseases aspects caused by parasitics and fungal in Ouagadougou (Burkina Faso)}




\section{ABSTRACT}

To determine the epidemiological and etiological aspects of lung diseases caused by parasitic and fungal in Ouagadougou, a prospective cross-sectional study was carried out in Ouagadougou Yalgado Ouédroago teaching hospital from November 2012 to May 2013 on 103 patients suspected. Parasitological, mycological and immunological tests were done on samples of lung washing fluid, pleural fluid, sputum and blood. After these tests $59.2 \%$ of patients have been confirmed parasite carrier and / or fungi in their pulmonary system with predominance of farmers (26.2\%). Men were the most affected with a sex ratio of 1.9. Among the medical history, there was a high frequency of HIV positive patients. Three parasites and 71 fungal strains were isolated out of which 4 types of fungal coinfections. These databases show that parasites and fungi cause non-specific lung disease clinically, radiological and endoscopic. Only the epidemiological and biological context can guide the diagnosis. The confirmation is given by direct detection or by serological indirect arguments. This allows obtaining an adapted treatment to the causative organism.

(C) 2016 International Formulae Group. All rights reserved.

Keywords: Lung diseases, parasites, fungi, Burkina Faso.

\section{INTRODUCTION}

Les parasitoses et les mycoses profondes occupent une place de plus en plus importante dans les pathologies pulmonaires infectieuses ces dernières décennies (Chabasse et al., 2009).

Bien qu'il soit difficile d'estimer leur incidence, selon l'Organisation Mondiale de la Santé (OMS), en 2005, 3 millions de personnes sont décédées de bronchopneumopathie chronique obstructive (Narain, 2013). Les agents pathogènes en cause demeurent méconnus dans 25 à $50 \%$ des cas en milieu hospitalier et $20 \%$ des pneumopathies surviennent en dehors de tout facteur favorisant identifié (e-Pilly TroP, 2012). En Côte d'Ivoire, $17,60 \%$ de parasitoses et de mycoses ont été trouvés dans les liquides d'aspirations bronchiques en Abidjan (Adou-Bryn et al., 1999). Ces taux varient en fonction de l'état de santé des populations et du niveau d'hygiène, mais est généralement plus élevé dans les pays en développement. Les agents pathogènes, à l'origine des infections pulmonaires chez l'homme provoquent, des symptômes d'une large gamme de sévérité se manifestant généralement d'une simple toux à des suppurations bronchiques nécessitant une prise en charge spécifique. Le diagnostic étiologique est basé sur la mise en évidence $\mathrm{du}$ parasite ou du mycète dans les expectorations, le lavage bronchoalvéolaire ou le liquide pleural. Le traitement spécifique à l'agent pathogène incriminé est le plus souvent suffisant pour entrainer la guérison (Bouree, 2013). Cette étude se propose de ressortir les aspects épidémiologiques, et étiologiques, des affections pulmonaires d'origines parasitaire et fongique chez les patients ayant consulté en milieu hospitalier à Ouagadougou, afin d'améliorer la prise en charge des patients suspects.

\section{MATERIEL METHODES}

La collecte des données et des échantillons biologiques a été réalisée dans le service de pneumo-phtisiologie du CHU Yalgado Ouédraogo de Ouagadougou et une clinique privée de la place reconnue dans la prise en charge des pathologies pulmonaires. Le service de parasitologie-mycologie dudit CHU nous a servi de cadre pour les manipulations biologiques. Pour trouver les informations, nous avons utilisé l'approche STEPSwise (Bonita et al., 2001) qui a été recommandée par l'Organisation Mondiale de la Santé (OMS), pour la région africaine, dans le cadre de la stratégie de la surveillance mondiale pour observer les tendances des 
maladies non transmissibles. Cette approche est un processus séquentiel qui commence par la collecte de données sur les comportements sanitaires clés à l'aide de questionnaires (Etape 1), puis à des mesures physiques simples (Etape 2) et ensuite seulement au prélèvement d'échantillons cliniques en vue d'une évaluation parasitologique, mycologique, et immunologique (Etape 3).

L'étape 1 et l'étape 2 se sont déroulées dans les services cliniques. Les fiches individuelles de collecte des données ont été remplies par le personnel (médecins, infirmiers) collaborant à l'étude. Les renseignements suivants ont été pris :

- Caractéristiques socio démographiques (âge, sexe, lieu de résidence, statut socio professionnel);

- Données épidémiologiques (consultations antérieures, traitements antérieurs, évolution antérieures des lésions, diabète, VIH, obésité, grossesse) ;

- Données cliniques (motif de consultation ou signes fonctionnels, signes physiques) ;

$$
\text { Aspects radiologique et }
$$

endoscopique pulmonaires.

L'étape 3 : le diagnostic biologique :

Toutes les techniques parasitologiques et mycologiques, de routines utilisées dans cette étude, respectent les protocoles d'exécution des examens réalisés au service de Parasitologie-Mycologie du Centre Hospitalier Universitaire Yalgado Ouedraogo et du fabriquant (ELI Tech MICROBIO) pour les examens immunologiques.

Le culot de centrifugation de chaque prélèvement a fait l'objet d'un examen macroscopique et microscopique direct. La coloration du Giemsa, de l'encre de chine été effectuées pour la mise en évidence des éléments constitutifs (larves, œufs, kystes) parasitaires.

Pour plus de fiabilité, le diagnostic biologique de parasitose pulmonaire dans cette étude état basé sur deux techniques de confirmation à partir du sérum du malade : Hémagglutination indirecte et le test d'agglutination sur lame des particules de latex. Les parasites recherchés sont: l'amibe, l'echinococcus, le toxoplasme.

Les différentes espèces de champignons et de levures pulmonaires étaient recherchées. Trois milieux ont été utilisés pour la culture mycologique :

- Le milieu SABOURAUD simple,

- Le milieu de SABOURAUD + chloramphénicol,

- Le milieu de SABOURAUD + chloramphénicol + actidione.

Les milieux ont été incubés entre $27^{\circ} \mathrm{C}$ et $37{ }^{\circ} \mathrm{C}$. L'identification des champignons était basée sur leur morphologie macroscopique et microscopique. La distinction de Candida albicans des autres Candida se fera par le test de pseudofilamentation avec le sérum de veau fœtal ou de lapin (test de BLASTESE) et de clamidosporulation avec le milieu Pomme de terre-Carotte-Bile (PCB).

\section{Considération éthique}

Elle a été au préalable respectée, par les autorisations d'enquête octroyées par la direction et les différents chefs de service du Centre Hospitalier Universitaire Yalgado Ouédraogo et par le directeur de la clinique privée. Aussi, nous avons demandé le consentement écrit des patients pour recueillir des renseignements sur leurs maladies.

\section{RESULTATS}

\section{Aspects épidémiologiques des parasitoses et} mycoses pulmonaires

Notre échantillon comprenait 103 patients dont $61(59,2 \%)$ étaient révélés positifs à l'examen parasitologique et/ou mycologique. Le sexe masculin était majoritaire 70,5\% avec un ratio de 1,9. L'âge moyen des patients était de 49,6 ans avec des extrêmes de 15 et 100 ans. La tranche d'âge de 30 à 45 ans était la plus touchée $(32,8 \%)$. Les 
cultivateurs $(26,2 \%)$ étaient majoritairement concernés. La majorité des patients $(65,5 \%)$ positifs dans cette étude vivaient dans la capitale à Ouagadougou. La répartition selon les antécédents médicaux, montrait que les patients séropositifs au VIH étaient les plus atteints $(21,3 \%)$ suivi des tabagiques $(6,5 \%)$. Les signes fonctionnels sont généralement accompagnés des signes physiques présentés dans les Tableaux 1 et 2 .

Cinquante-neuf (59) patients ont présenté des images radiologiques anormales. Le plus souvent, il s'agit des syndromes interstitiels bilatéral ou alvéolaire, de broncho pneumopathie et d'opacité. Les abcès pulmonaires et les opacités étaient les seuls signes radiologiques rencontrés au cours des parasitoses pulmonaires avec des fréquences respectives de $66,6 \%$ et $33,4 \%$. A l'examen endoscopique, les inflammations et les suppurations bronchiques étaient les lésions les plus fréquentes avec $23,8 \%$ partout.

\section{Aspects étiologiques}

Le Tableau 3 montre la fréquence des groupes et espèces parasites et mycètes

Nous avions noté 4 cas d'associations fongiques.

L'association entre Pneumocystis jerovecii et Candida sp. était mise en évidence 4 fois ;

L'association entre A. niger, $A$. fumigatus, A. flavus, A. nidulans et Candida $s p$. était mise en évidence 1 fois ;

L'association entre A. fumigatus et $C$. albicans était mise en évidence 1 fois;

L'association entre $A$. niger et $A$. nidulans était mise en évidence 1 fois.

Tableau 1 : Fréquence des signes fonctionnels.

\begin{tabular}{lccc}
\hline Signes fonctionnels & $\begin{array}{c}\text { Nb de patients } \\
\text { Examinés }\end{array}$ & $\begin{array}{c}\text { Nb de patients } \\
\text { Infectés }\end{array}$ & $\begin{array}{c}\text { Pourcentage (\%) de } \\
\text { patients Infectés }\end{array}$ \\
\hline Toux & 80 & 48 & 60 \\
Dyspnée & 59 & 31 & 52,5 \\
Douleur thoracique & 38 & 27 & 71 \\
\hline
\end{tabular}

Tableau 2 : Fréquence des signes physiques.

\begin{tabular}{lccc}
\hline Signes physiques & $\begin{array}{c}\text { Nb de patients } \\
\text { examinés }\end{array}$ & $\begin{array}{c}\text { Nb de patients } \\
\text { Infectés }\end{array}$ & $\begin{array}{c}\text { Pourcentage (\%) de } \\
\text { patients infectés }\end{array}$ \\
\hline Suppuration pulmonaire & 30 & 18 & 60 \\
Hémoptysie & 21 & 14 & 66 \\
Absence de signe & 32 & 18 & 56,3 \\
Autre* & 20 & 11 & 55 \\
Totale & 103 & 61 & 59,2 \\
\hline$\square \quad$ Amaigrissement, Altération de l'Etat Général, Insuffisance rénale, Hépatomégalie, Fièvre, Anémie, Ascite, \\
Cardiopathie, Crise convulsive.
\end{tabular}


Tableau 3 : Fréquence des groupes et espèces parasites et mycètes.

\begin{tabular}{|c|c|c|}
\hline Parasites/Mycètes & Nombre d'isolement & Fréquence (\%) \\
\hline Parasites & 3 & 4 \\
\hline Entamoeba histolytica & 3 & 4 \\
\hline Mycètes & 71 & 96 \\
\hline Mycètes filamenteux & 38 & 51,4 \\
\hline Aspergillus fumigatus & 14 & 19 \\
\hline Aspergillus flavus & 8 & 10,9 \\
\hline Aspergillus niger & 6 & 8,1 \\
\hline Aspergillus nidulans & 5 & 6,7 \\
\hline Aspergillus sp. & 3 & 4 \\
\hline Aspergillus versicolor & 2 & 2,7 \\
\hline Mycètes levurifomes & 28 & 37,9 \\
\hline Candida sp. & 17 & 22,9 \\
\hline Candida albicans & 10 & 13,6 \\
\hline Cryptococcus neoformans & 1 & 1,4 \\
\hline Mycète inclassé & 5 & 6,7 \\
\hline Pneumocystis jirovecii & 5 & 6,7 \\
\hline Total & 74 & 100 \\
\hline
\end{tabular}

\section{DISCUSSION}

La prévalence de $59,2 \%$ de patients atteints de parasitose et de mycose pulmonaire, montre que ces infections qui ne pouvaient qu'être suspectées cliniquement sont devenues une réalité en pratique pneumologique au Burkina-Faso. Toutefois, les patients, dans le contexte de faibles revenus et de l'inaccessibilité géographique aux structures sanitaires, ne consultent que tardivement lorsque leur état est devenu très critique (Zoubga et al., 2000).

Les hommes étaient les plus atteints, à cause de leurs professions qui les exposent aux dangers de la pollution atmosphérique, à l'astreinte physique mais aussi le risque d'alcoolisme et de tabagisme dont une grande partie d'entre eux encourent. Des études précédentes sur les pathologies pulmonaires en milieu hospitalier Burkinabè, ayant inclus d'autres étiologies que parasitaires et fongiques, ont fait ce même constat (Ouedraogo et al., 2000a ; Ouedraogo et al., 2000b; Ouedraogo et al., 2001;
Ouedraogo et al., 2004; Chabasse et al., 2009)

Les infections parasitaires et fongiques sont peu fréquentes chez les jeunes enfants mais très présentes chez les adultes. Nos résultats montrent que la maladie touche particulièrement les bras valides de la population, d'où les conséquences lourdes sur le développement économique du pays. Nous avons trouvé une prédominance notoire des habitants vivant en zone urbaine notamment la capitale Ouagadougou. Les champignons sont des germes ubiquitaire. La pollution biologique induite par des taux anormaux d'allergènes de microbes ou de spores fongiques est en augmentation et semblent souvent exacerbés par les polluants urbains, routiers et de l'industrie (Mohamed et al., 2002). Sur le plan professionnel, les cultivateurs étaient les plus représentés suivis des fonctionnaires et des ménagères. En Afrique de l'ouest, l'agriculture et l'élevage sont l'activité quotidienne des pauvres, souvent pratiqués par les mêmes personnes. 
Leur risque de contamination par certains parasites comme $P$. jirovecii et les aspergillus retrouvés dans les fentes des pigeons, les foins moisis et la poussière des céréales est élevé (Quatresous, 2011). De nombreux pathogènes zoonotiques, sont transmis à l'homme par contact direct ou indirect (vecteurs). Cette couche socioprofessionnelle de niveau d'instruction faible, ignore ou n'a pas accès aux règles d'hygiène corporelle nécessaire à l'éviction par exemple de l'amibiase et un mauvais terrain immunitaire sont en cause. L'immunodépression due au VIH et le tabagisme sont les facteurs de risque les plus incriminés dans les parasitoses et mycoses pulmonaires dans l'étude.

Selon l'OMS, les combustibles et les méthodes de cuisson produisent de niveaux élevés de pollution de l'air dans les logements, mettent en jeu une multitude de polluants nocifs pour la santé, notamment de fines particules de suie qui pénètrent dans les poumons en profondeur et les fragilisent. Dans les habitations insuffisamment ventilées, la teneur en particules fines dans la fumée domestique peut atteindre une concentration 100 fois supérieure aux niveaux acceptables (OMS, 2014).

Le biologiste est confronté au développement inattendu des parasites dans tout l'organisme lors de déficience des défenses immunitaires. Les aspects cliniques classiquement observés peuvent être totalement modifiés. Une grande prudence et beaucoup de vigilance sont alors nécessaires. La toux, la dyspnée, les douleurs thoraciques étaient les motifs les plus fréquents de consultations des patients, accompagné souvent des hémoptysies, voir des suppurations au cours des parasitoses et mycoses pulmonaires en milieu hospitalier à Ouagadougou. Il convient aussi de noter, par comparaison, que cette fréquence des hémoptysies (66\%) est inférieure aux 50\% rapportée par Saghrouni et al. (2011) en Tunisie chez des patients neutropéniques. Mais ces auteurs ont spécifiquement ciblé le genre aspergillus et n'ont pas étendu leur diagnostic étiologique aux autres champignons et parasites pulmonaires.

Autrefois, le diagnostic des parasitoses qui était basé sur les biopsies pulmonaires, s'effectue actuellement sur la mise en évidence des formes végétatives et des kystes parasitaire et fongique dans le liquide de lavage bronchoalvéolaire (Magne et al., 2009). Les techniques de sérologie parasitaire permettent une confirmation du diagnostic clinique, radiologique et parfois des examens des pièces biopsiques. Entamoeba histolytica était la seule espèce parasitaire rencontrée dans notre étude avec une fréquence de 4\% des identifications. Adou-Bryn et al. (1999) ont déjà trouvé une fréquence similaire de $3,85 \%$ dans les liquides d'aspirations bronchiques à Abidjan en Côte d'Ivoire.

En ce qui concerne les champignons, nous les avons rencontrés dans $96 \%$ des identifications. Ce taux est proche des $96,15 \%$ trouvé par Adou-Bryn et al. (1999) et Sudfeld et al. (2010). L'Aspergillus $(51,4 \%)$ est le seul champignon filamenteux rencontré dans l'étude. L'espèce majoritairement rencontrée l'Aspergillus fumigatus avec $16,3 \%$ des patients colonisés. Dans la littérature, sa fréquence varie de 6-56\% (Pihet et al., 2009). Nous nous trouvons donc dans la moyenne. Il est intéressant de remarquer que cette valeur varie selon les pays : $36 \%$ aux Etats Unis (Blyth et al., 2010), 25\% au Danemark, 45\% en Allemagne (Bouchara et al., 2009). A Ouagadougou le taux d'Aspergillus non fumigatus s'élève à $35,4 \%$ dans les études publiées ce taux est de 10,4\% (Paugam et al., 2010) et s'élève jusqu'à $26,1 \%$ dans l'étude menée par Sudfeld et al. (2010).

Parmi les levures, le genre Candida représentait $22,9 \%$ des isolements. Cette valeur est inférieure aux $46,1 \%$ trouvés par Adou-Bryn et al. (1999), mais supérieure aux $18,75 \%$ rapportés chez les immunodéprimés par Silva (2010). Candida albicans est l'espèce de levure la plus identifiée avec $13.6 \%$. En réanimation, des prélèvements 
positifs à Candida sont fréquents en dehors de toute immunodépression. La physiopathologie de ces « infections respiratoires basses à Candida » implique probablement une dissémination bronchique de haut en bas à partir d'une colonisation trachéobronchique chez des patients intubés, éventuellement alcooliques ou diabétiques. Cette colonisation bronchique à Candida peut être aussi favorisée par une antibiothérapie antibactérienne (Azoulay et al., 2001).

Cryptococcus neoformans est rare au cours d'une infection pulmonaire. Alors que Pneumocystis jirovecii est fréquemment rapporté en particulier chez les immunodéprimés avec un taux qui varie selon les auteurs 23,6\% au Cameroun (Fewou et al., 2004). Dans notre série, il affecte 6,7 des patients.

La variation des résultats suscite des réflexions sur l'influence de l'âge, des conditions climatiques et géographiques des champignons (Bakare et al., 2003). Le nombre et la nature des cultures, les temps d'incubations, la température et le manque de standardisation des méthodes d'examens mycologiques sont également en causes (JeanPhillippe et al., 2009).

\section{Conclusion}

Les parasites et les champignons provoquent une pathologie pulmonaire non spécifique sur le plan clinique. Le contexte épidémiologique et biologique permet d'orienter le diagnostic. La confirmation est apportée par la mise en évidence directe ou par des arguments indirects sérologiques. Ce qui permet d'obtenir une guérison par un traitement adapté au germe en cause. La détérioration de la fonction respiratoire, associé à l'intensité de colonisation et de surinfection broncho-pulmonaire conditionne le pronostique des patients. Dans notre contexte, la colonisation par la flore fongique est bien moins connue que la colonisation bactérienne. Cette étude permet de situer le traitement probabiliste pour une meilleure prise en charge des patients.

\section{CONFLIT D'INTERETS}

Il n'existe pas de conflit d'intérêt pour cet article.

\section{CONTRIBUTIONS DES AUTEURS}

OAM a été le principal investigateur ; ZA a dirigé l'aspect technique directeur technique des travaux de la recherche ; $\mathrm{MH}$ a participé à la rédaction de l'article; SA a été conseiller externe de la recherche; TAS à diriger les travaux de recherche; OM à Contribuer à la sélection des cas clinique pendant la recherche.

\section{REFERENCES}

Adou-Bryn KD, Ouhon J, Assoumou A, Kassi EA, Kone M, Therizol-Ferly M. 1999. Champignons et parasites isoles à l'examen de 142 liquides d'aspiration bronchique à Abidjan. Méd. Afr. Noire, 46: 363-365.

Azoulay E, Limal N, Mayaud C, Schlemmer B. 2001. Prélèvement pulmonaire positif à Candida : infection nosocomiale ou colonisation. Réanimation, 10: 8-323.

Bakare N, Rickerts V, Bargon J, Just-Nübling G. 2003. Prevalence of Aspergillus fumigatus and other fungal species in the sputum of adult patients with cystic fibrosis. Med Mycol., 46 (1-2): 19-23.

Blyth C, Middleton P, Harun A, Sorrell T, Meyer W, Chen S. 2010. Clinical associations and prevalence of Scedosporium spp. in Autralian cystic fibrosis patients: identification of nouvel risk factor? Medical Mycology, 48: 37-44.

Bonita R, de Courten M, Dwyer T, Jamrozik K, Winkelmann R. 2001. Surveillance des facteurs de risque des maladies non transmissibles : l'approche STEPwise de l'OMS. Résumé. Genève, Organisation mondiale de la Santé. 
http://www.who.int/ncd/surveillance/su rveillance_publications.htm

Bouchara JP, Yi Hsieh H, Croquefer S, Barton R, Marchais V, Pihet M, Chang T. 2009. Development of an oligonucleotide array for direct detection of fungi in sputum samples from patients with cystic fibrosis. Journal of Clinical Microbiology, 1: 142-152.

Bouree P. 2013. Parasitoses pulmonaires. EMC-Pneumologie, 10: 2-8.

Chabasse D, Pihet M, Bouchara JP. 2009. Émergence de nouveaux champignons pathogènes en médecine. Revue Francophone des Laboratoires, 416: 71-86.

e-Pilly TroP. 2012. Maladies infectieuses tropicales, $\quad$ p. 215-240. http://www.infectiologie.com/site/livres .php.

Fewou A, Essame O, Mfou'ou Azo'o F, Afane Ze E, Ndom P, Gasser B. 2004. L'atteinte pulmonaire au cours du SIDA : aspects histo-pathologiques en milieu Camerounais. Résultats préliminaires. Méd. Afr. Noire, 51(1): 55-59.

Jean-Phillippe B, Hsin Yi H, Sabine C, Richard B, Veronique M, Marc P, Tsung CC. 2009. Development of an Oligonucleotide Array for Direct Detection of Fungi in Sputum Samples from Patients with Cystic Fibrosis. $J$ Clin Microbiol., 47(1): 142-152

Mohamed L, Karine L, Jean-Pierre B. 2002. Synergie entre pollens et polluants chimiques de l'air : les risques croisés. Environnement, Risques \& Santé, 1: 42-9.

Magne D, Angoulvant A, Botterel F, BougesMichel C, Bougnoux ME, Bouré P. 2009. Réseau francilien : bilan de cinq années de surveillance 2003-2007. J Mycol Med., 19: 290-293.

Narain JP. 2013. Prevention and control of non-communicable diseases:
Guidelines for primary health care in low-resource settings (World Health Organization, Geneva, Switzerland. 2012). Indian J Med Res., 138: 10341037.

Ouedraogo M, Zoubga ZA, Badoum G, Ouedraogo SM, Ouedraogo G, Bambara M, Thiombano PN, Koshinga BA, Drabo YJ. 2004. Morbi-mortalité liée aux maladies respiratoires chez les malades infectés par le VIH à Ouagadougou. Méd. Afr. Noire, 51: 456-458.

Ouedraogo M, Boncoungou K, Ouedraogo SM, Lougue C, Cisse R, Birba E, Bambara M, Koshinga BA, Drabo YJ. 2001. Miliaire tuberculeuse bacillifére : à propos de 44 cas au CHU YO/Burkina Faso. Méd. d'Afr. Noire, 48: 181-183.

Ouedraogo M, Ky C, Ouedraogo SM, Zoubga AZ, Badoum G, Zigani A, Bambara M, Ouedraogo G, Compaore I, Auregan G, Kabore J. 2000a. Aspects épidémiocliniques de la pleurésie sero-fibrineuse au Centre Hospitalier National Yalgado Ouedraogo. Méd. d'Afr. Noire, 47: 387389.

Ouedraogo M, Ouedraogo G, Ouedraogo SM, Zoubga ZA, Bambara M, Ky C, Badoum G, Zigani A, Some L, Drabo YJ. 2000b. Aspects épidémiologiques et cliniques des miliaires tuberculeuses au Burkina Faso : A propos de 93 cas. Méd. d'Afr. Noire, 47: 420-422.

OMS: Organisation Mondiale de la santé. 2014. Pollution de l'air à l'intérieur des habitations et la santé. Aide-mémoire No292. DOI : http://www.who.int/ mediacentre/factsheets/fs292/fr/ consulté le 2 septembre 2015.

Paugam A, Baixench MT, Demazes-Dufeu N, Burgel PR, Sauter E, Kanaan R, Dusser D, Dupouy-Camet J, Hubert D. 2010. Characteristic and consequences of airways colonization by filamentous fungi in 201 adult patients with cystic 
fibrisis in France. Medical Mycology., 48: $32-36$.

Pihet M, Carrere J, Cimon B, Chabasse D, Delhaes L, Symoens F, Bouchara JP. 2009. Occurrence and relevance of filamentous fungi in respiratory secretion of patients with cystic fibrosis-a review. Medical Mycology, 47: 387-397.

Quatresous N. 2011. Aspergillose humaine: Épidémiologie, diagnostic biologique, contrôle. Thèse Pharmacie, Université de Limoges., p. 136. DOI: http://hdl.handle.net/10068/857196 consulté le 5 février 2013.

Saghrouni F, Ben Youssef Y, Gheith S, Bouabid Z, Ben Abdeljelil J, Khammari I, Fathallah A, Khlif A, Ben Saïd M. 2011. Twenty-nine cases of invasive aspergillosis in neutropenic patients. Méd. Mal. Infect., 41: 657662.
Silva RF. 2010. Fungal infections in immunocompromised patients. J. Bras. Pneumol., 36: 142-147.

Sudfeld C, Desenbrook E, Merz W, Carrol K, Boyle M. 2010. Prevalence and risque factors for recevery of filamentous fungi in individuals with cystic fibrosis. Journal of cystic fibrosis, 9: 110-116.

Zoubga AZ, Ouédraogo M, Boncoungou K, KI C, Ouedraogo SM, Ouédraogo G, bambara M, Birba E, Millogo GRC, Some L, Drabo YJ. 2000. Aspects épidémiologiques, cliniques et thérapeutiques des pneumopathies aiguës bactériennes dans le service de pneumologie du centre hospitalier national Sanou Souro de BoboDioulasso. Méd. d'Afr. Noire, 47(11): 471-472. 